\title{
THE PRACTICALITY OF LATIN
}

\author{
Edward G. Voss
}

The plea by Dr. Kostermans asks why we continue to require Latin diagnoses to validate new names and states no good reason for the requirement. Instead, he attacks an assortment of "straw men" like support from an Old Guard (not defined) and a notion that Latin somehow adds prestige or scientific "image," as well as noting the indisputable (but irrelevant) fact that professors no longer lecture in Latin.

I am compelled to state the sound, practical reasons why Latin should remain compulsory:

(1) The fact that use of Latin is no longer widespread in speaking and writing is actually in its favor. As a "dead" language it is not changing as are modern languages, in which meanings of words evolve, slang becomes acceptable or else dies out, and usage varies in different regions. "Botanical Latin," so usefully codified by William Stearn, has a relatively stable basic vocabulary; Dr. Kostermans admits that even "language-faulty diagnoses" tend to be good enough to be understood-and that is exactly the point. If English were to become acceptable for validating names, I would have to know whether it was a British or American author who describes a spore or seed as "football-shaped" or a fruit as "ballbat-shaped." What does a flower look like that resembles a "bluebell"? Use of a modern language would unnecessarily encourage wider use of current vocabulary fads, contemporary (and perhaps ephemeral) imagery, vernacular names, and geographic linguistic biases.

(2) The Latin requirement is immensely important in recognizing deliberate intention to publish new names. Literature published before that requirement is full of inadvertent publication of new names, e.g., in travel narratives, the format of keys and tables, or ecological papers. When all that is required is two or more descriptive words in the language used by the writer, there can be a tremendous problem identifying new names. Editors and publishers (who may indeed also be the authors - or who may know nothing of taxonomy) cannot be relied upon to refuse to print papers that fail to uphold minimal nomenclatural standards. However, omission of the necessary Latin automatically helps to eliminate consideration of substandard work. Dr. Kostermans declares that poor papers in local languages often do not reach the scientific community and that this is perhaps good "as their quality is usually below par." Unfortunately, in nomenclature we can not overlook work of which the quality is below par, no matter what the language (nor whether we can agree on quality). If a name has been published with Latin diagnosis and the other requirements, it must be taken into account for purposes of homonymy, indexing, and so forth. Minimal explicit requirements, like Latin and designation of a type, are an objective and non-political means of encouraging and recognizing serious intentions and competent scholarship.

A proposal (Art. 36, Prop. A) to allow either English or Latin was soundly defeated at the Berlin Congress in 1987 , the preliminary mail vote having been against it approximately $4: 1$. I hope that vote reflects the opinion of taxonomists generally.

1 Herbarium, North University Bldg., University of Michigan, Ann Arbor, MI 48109-1057, U.S.A. 